\title{
Optical probing of MgZnO/ZnO heterointerface confinement potential energy levels
}

Cite as: Appl. Phys. Lett. 106, 082102 (2015); https://doi.org/10.1063/1.4913313

Submitted: 25 December 2014 . Accepted: 10 February 2015. Published Online: 23 February 2015

V. V. Solovyev, A. B. Van'kov, I. V. Kukushkin, J. Falson, D. Zhang, D. Maryenko, Y. Kozuka, A. Tsukazaki, J. H. Smet, and M. Kawasaki
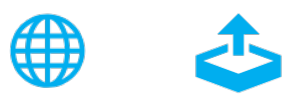

\section{ARTICLES YOU MAY BE INTERESTED IN}

Electron scattering times in $\mathrm{ZnO}$ based polar heterostructures

Applied Physics Letters 107, 082102 (2015); https://doi.org/10.1063/1.4929381

Polarization-induced two-dimensional electron gases in $\mathrm{ZnMgO} / \mathrm{ZnO}$ heterostructures

Applied Physics Letters 93, 202104 (2008); https://doi.org/10.1063/1.3028338

Challenges and opportunities of ZnO-related single crystalline heterostructures

Applied Physics Reviews 1, 011303 (2014); https://doi.org/10.1063/1.4853535

Lock-in Amplifiers Find out more today

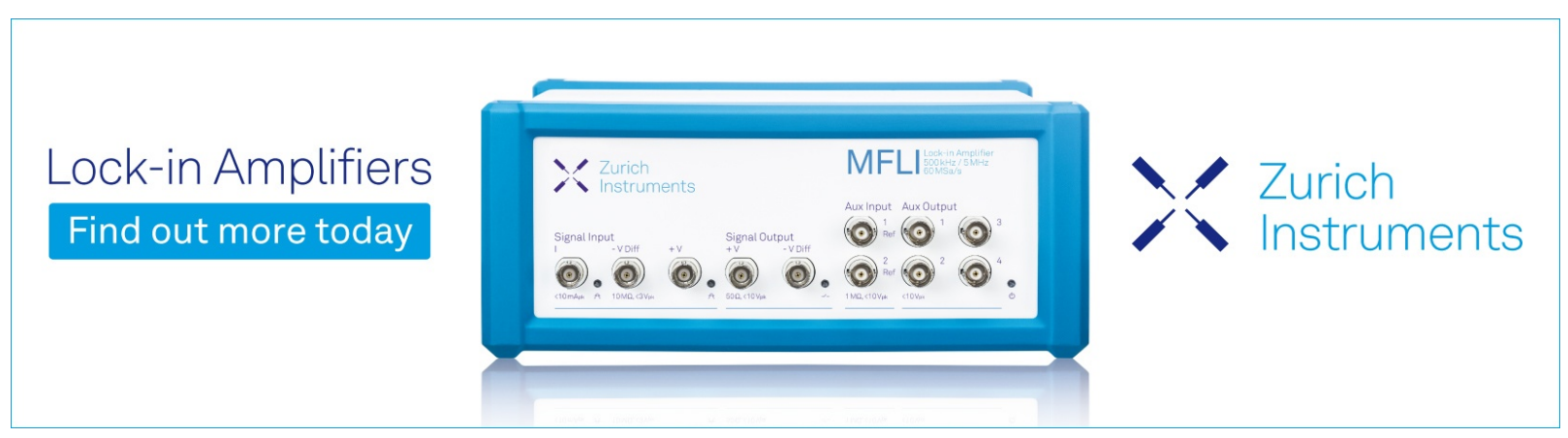




\title{
Optical probing of $\mathrm{MgZnO} / \mathrm{ZnO}$ heterointerface confinement potential energy levels
}

\author{
V. V. Solovyev, ${ }^{1,2}$ A. B. Van'kov, ${ }^{1,2}$ I. V. Kukushkin, ${ }^{1,2}$ J. Falson, ${ }^{3}$ D. Zhang, ${ }^{2}$ D. Maryenko, ${ }^{4}$ \\ Y. Kozuka, ${ }^{3}$ A. Tsukazaki, ${ }^{5,6}$ J. H. Smet, ${ }^{2}$ and M. Kawasaki ${ }^{3,4}$ \\ ${ }^{1}$ Institute of Solid State Physics, RAS, Chernogolovka 142432, Russia \\ ${ }^{2}$ Max-Planck-Institut für Festkörperforschung, Heisenbergstrasse 1, D-70569 Stuttgart, Germany \\ ${ }^{3}$ Department of Applied Physics and Quantum-Phase Electronics Center (QPEC), University of Tokyo, \\ Tokyo 113-8656, Japan \\ ${ }^{4}$ RIKEN Center for Emergent Matter Science (CEMS), Wako 351-0198, Japan \\ ${ }^{5}$ Institute for Materials Research, Tohoku University, Sendai 980-8577, Japan \\ ${ }^{6}$ PRESTO, Japan Science and Technology Agency (JST), Tokyo 102-0075, Japan
}

(Received 25 December 2014; accepted 10 February 2015; published online 23 February 2015)

Low-temperature photoluminescence and reflectance measurements were employed to study the optical transitions present in two-dimensional electron systems confined at $\mathrm{Mg}_{x} \mathrm{Zn}_{1-x} \mathrm{O} / \mathrm{ZnO}$ heterojunctions. Transitions involving A- and B-holes and electrons from the two lowest subbands formed within the confinement potential are detected. In the studied density range of $2.0-6.5 \times 10^{11} \mathrm{~cm}^{-2}$, the inter-subband splitting is measured and the first excited electron subband is shown to be empty of electrons. (C) 2015 AIP Publishing LLC. [http://dx.doi.org/10.1063/1.4913313]

Wide direct band gap semiconductor materials such as $\mathrm{ZnO}, \mathrm{GaN}$, and $\mathrm{BN}$ offer improved performance for future photonic and electronic power devices due to higher optical transition energies, electric breakdown field, and saturation velocity compared with $\mathrm{Si}$ and GaAs. ${ }^{1}$ In the case of $\mathrm{ZnO}$, progress in crystal growth techniques now enables the development of high quality $\mathrm{Mg}_{x} \mathrm{Zn}_{1-x} \mathrm{O} / \mathrm{ZnO}$ heterojunctions (HJ) which host high mobility two-dimensional electron systems (2DES). ${ }^{2-5}$ In contrast to the $\mathrm{Al}_{x} \mathrm{Ga}_{1-x} \mathrm{As} / \mathrm{GaAs}$ $(x>0.2)$ heterointerface where the presence of a large barrier height in combination with modulation doping determines both the energy band diagram and electron distribution between different quantized subbands of the $2 \mathrm{DES},{ }^{6,7}$ it is the discontinuity in internal polarization at the $\mathrm{Mg}_{x} \mathrm{Zn}_{1-x} \mathrm{O} /$ $\mathrm{ZnO}$ heterointerface that naturally induces a $2 \mathrm{DES}^{8}{ }^{8}$ The charge density $(n)$ is therefore determined by the discontinuity in electric field, which is a monotonic function of $\mathrm{Mg}$-fraction. ${ }^{2}$ However, the barrier height too grows linearly with $\mathrm{Mg}$-content $x$. It is therefore unclear a priori what electron distribution is realized at the $\mathrm{HJ}$ for each particular value of $\mathrm{Mg}$.

Here, we investigate the energy levels formed at the $\mathrm{Mg}_{x} \mathrm{Zn}_{1-x} \mathrm{O} / \mathrm{ZnO} \mathrm{HJ}$ through optical measurements. A series of $2 \mathrm{DES}$ covering the electron density range from 2.0 to $6.5 \times 10^{11} \mathrm{~cm}^{-2}$ with varying $x=0.014-0.045$ were studied by means of photoluminescence (PL) and reflectance measurements at temperatures of $0.5 \mathrm{~K}$ and in perpendicular magnetic fields up to $B=14 \mathrm{~T}$. A number of optical transitions show periodic oscillations in energy with $B$ and can be associated with the Landau level spectrum of a 2DES. They involve the two lowest subbands of the confinement potential in the conduction band as well as two branches of the valence-bands. It is possible to quantify the splitting of the two lowest electron subbands. It grows monotonically with density. For all heterostructures, only the lowest electron subband is occupied. Calculations of the confinement potential suggest that the full-width-half-maximum of the wavefunction is less than $10 \mathrm{~nm}$.

The $\mathrm{Mg}_{x} \mathrm{Zn}_{1-x} \mathrm{O} / \mathrm{ZnO}$ heterostructures were grown by ozone-molecular beam epitaxy. ${ }^{3}$ Low temperature $(T=0.5 \mathrm{~K})$ transport measurements (not shown) as well as the optical experiments described below yield the electron mobility, $\mu_{t}$, and density, $n_{t}$ and $n_{o}$, respectively (see Table I). These samples are much lower in density and higher in quality than those of previous optical studies. ${ }^{9,10}$ Photoluminescence and reflectance measurements were conducted in a two-fiber geometry. PL spectra were recorded by exciting the sample with the continuous-wave output of a $325 \mathrm{~nm}$ He-Cd laser, while the light from a commercial $365 \mathrm{~nm}$ light emitting diode was employed to detect reflectance spectra. The excitation power density was below $30 \mu \mathrm{W} / \mathrm{mm}^{2}$. PL and reflectance spectra were obtained in one cooldown using a Monospec spectrometer with a linear dispersion of $5 \AA / \mathrm{mm}$ and a spectral resolution of $0.3 \AA$.

Both the PL and reflectance spectra contain a multitude of features mostly stemming from optical transitions in bulk $\mathrm{ZnO}$ and $\mathrm{Mg}_{x} \mathrm{Zn}_{1-x} \mathrm{O}$. We concentrate on features near the $\mathrm{ZnO}$ fundamental gap energy and investigate their origin. They are marked with triangles, circles, and squares in Fig. 1. A hallmark of feature associated with $2 \mathrm{D}$ electrons is oscillating behavior periodic in $1 / B$, as a result of the sawtooth motion of the chemical potential in a Landau quantized 2DES of fixed density. All features highlighted in Fig. 1

TABLE I. The electron mobility $\mu_{t}$ and density from transport $\left(n_{t}\right)$ and optical studies $\left(n_{o}\right)$ of the investigated samples.

\begin{tabular}{lccc}
\hline \hline Sample & $n_{t}\left(10^{11} \mathrm{~cm}^{-2}\right)$ & $n_{o}\left(10^{11} \mathrm{~cm}^{-2}\right)$ & $\mu_{t}\left(10^{3} \mathrm{~cm}^{2} / \mathrm{V} \cdot \mathrm{s}\right)$ \\
\hline 282 & 1.9 & 2.0 & 270 \\
244 & 2.3 & 2.3 & 400 \\
202 & 3.5 & 3.6 & 300 \\
302 & 6.2 & 6.5 & 160 \\
\hline \hline
\end{tabular}




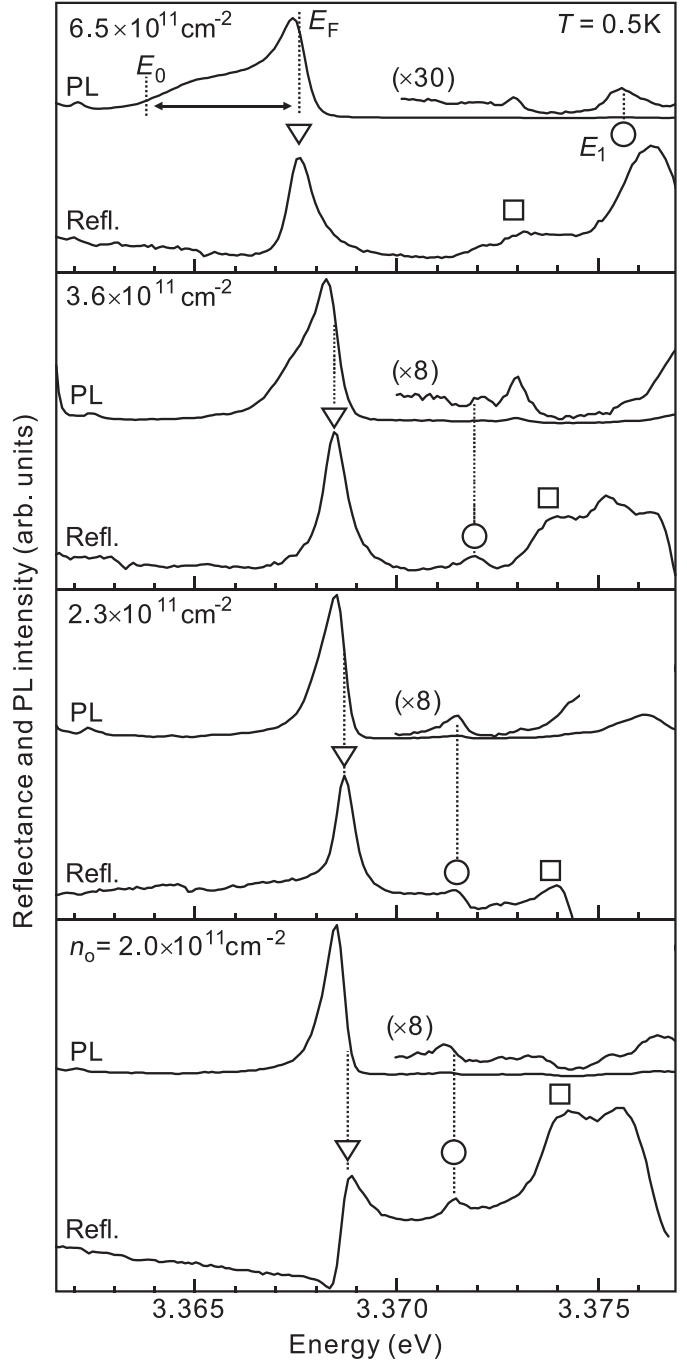

FIG. 1. Reflectance (bottom) and photoluminescence (top) spectra for the samples in Table I with density $n_{o}$. The evolution with density of three 2DES-related optical transitions in the reflectance are marked with triangles, squares, and circles. Transitions marked by circles and triangles are correlated with PL features (dotted lines).

show such periodicity. In Fig. 2 , the $B$-field dependence of the PL intensity recorded on sample $302\left(n_{o}=6.5 \times 10^{11} \mathrm{~cm}^{-2}\right)$ at the fixed energies of $3.3675 \mathrm{eV}$ and $3.3755 \mathrm{eV}$ is shown. These energies correspond to the triangles and circles in Fig. 1, and features are observed in both PL and reflectance. The most prominent oscillations in intensity occur at the $3.3675 \mathrm{eV}$ PLband. The locations of the intensity minima correspond closely to integer fillings $\nu=1,2,3, \ldots\left(\nu=\frac{h n}{e B}\right)$ of Landau levels. We therefore associate them with PL recombination of $2 \mathrm{D}$ electrons at the heterointerface. The intensity oscillations of the spectral line at $3.3755 \mathrm{eV}$ are in anti-phase with the lower energy band at $3.3675 \mathrm{eV}$. The reasons will be discussed later. The optical oscillations ${ }^{11}$ allow the quantification of the charge density $\left(n_{o}\right)$ under illumination, which is slightly enhanced in comparison with values from transport data (see Table I). Fig. 3 displays examples of the $B$-dependent behavior in the reflectance spectrum of sample 244. The center-of-mass energy values of the three spectral features marked in Fig. 1 with a triangle, circle, and square plotted as a function of $B$. Again, the observed maxima correlate with integer fillings.

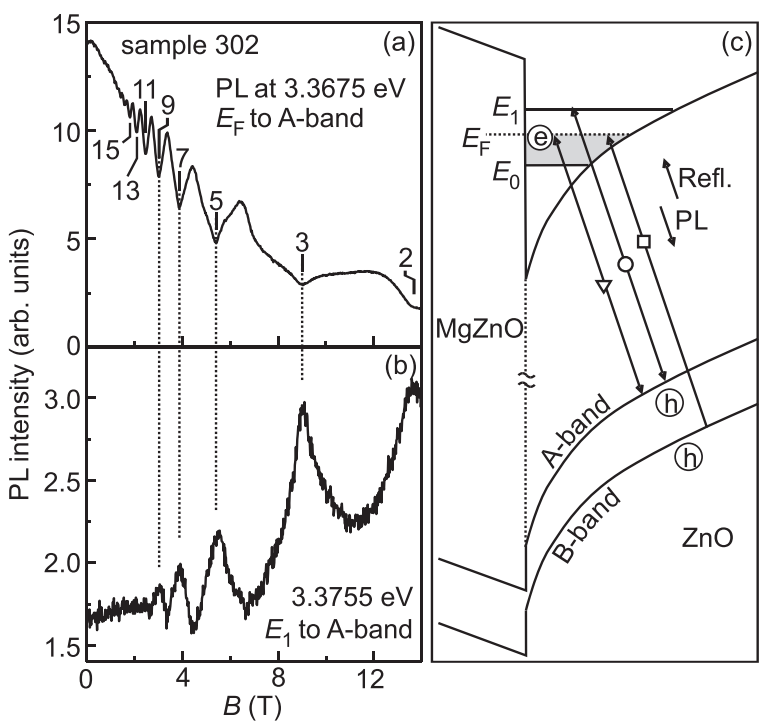

FIG. 2. (a) Magnetic field dependence of the PL-signal at a photon energy of $3.3675 \mathrm{eV}$ corresponding to $E_{F}$. Features associated with integer filling of the Landau levels are marked and enable the extraction of the carrier density: $n_{o}=6.5 \times 10^{11} \mathrm{~cm}^{-2}$. (b) Same for photon energy $3.3755 \mathrm{eV}$, corresponding to line $E_{1}$ in Fig. 1. The oscillations at this energy are synchronous (but anti-phase) with oscillations at $E_{F}$, i.e., $3.3675 \mathrm{eV}$. (c) Schematic drawing of the optical transitions between the electron subbands and two branches of the valence band. Upward and downward arrows depict transitions active in reflectance and PL experiments, respectively.

The lowest energy line in the reflectance spectra, marked by triangles in Fig. 1, is attributed to optical transitions from the top valence band state (A-band) to just above the Fermi energy $E_{F}$ of the electrons in the lowest subband of the conduction band (see Fig. 2(c)). This assignment is supported through a comparison with the PL spectrum, which is plotted above each reflectance spectrum. The line coincides with the abrupt termination of the PL-band of highest intensity. The energy span of the PL-band for the

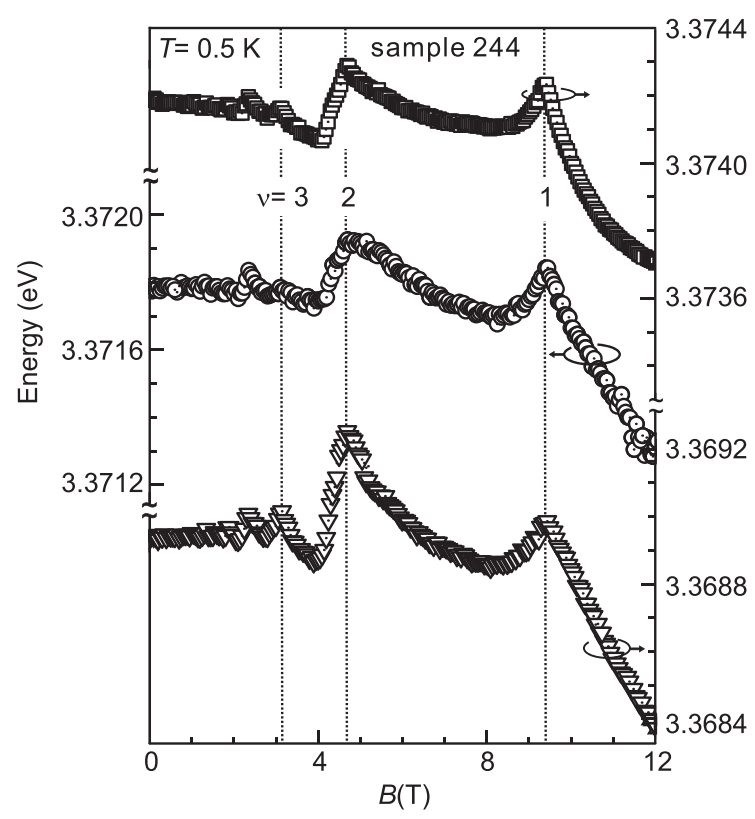

FIG. 3. Magnetic field dependence of the center-of-mass of the three features in the reflectance spectrum of sample $244\left(n_{o}=2.3 \times 10^{11} \mathrm{~cm}^{-2}\right)$ originating from $2 \mathrm{D}$ electrons. They are marked by a triangle, circle, and square in Fig. 1. 
highest density sample is marked by a double arrow. It grows with density, so that it can be related to the Fermi energy of the $2 \mathrm{DES} .{ }^{12}$ The high energy edge experiences a red-shift at higher densities presumably due to the dependence of the electron-hole energy separation on band bending near the HJ. Every interband transition involves the creation (for reflectance) or annihilation (for PL) of a valence band hole located at some distance from the HJ. The total energy of a hole reaches a minimum at some distance as it experiences the Coulombic attraction to the 2DES, but also the repulsive action from the built-in electric field near the HJ. This last energy term grows with the electron density resulting in a larger separation of the hole from the 2D channel. Hence, the optical transitions from and to the lowest electron subband redshift with density.

The assignment of both higher energy spectral lines in the reflectance spectra is more complex. Higher lying electron subbands at the heterointerface may possibly contribute. Alternatively or in addition, these features may be associated with the close energetic proximity of the heavy and light hole branches (A- and $\mathrm{B}$-branches) in the $\mathrm{ZnO}$ valence band. The crystal-field splitting is only about $5 \mathrm{meV} \cdot{ }^{13-15}$ The density dependence of the spectral features is summarized in Fig. 4(a) using the same symbols as in Fig. 1. There is an apparent correlation between the previously identified feature at $E_{F}$ (triangles) with the feature marked by squares. A monotonic decrease in energy is observed for both with increasing density. They are offset by $5.5 \mathrm{meV}$. Hence, we can attribute them to optical transitions from the crystal field split A- and B-valence bands to the lowest electronic subband of the 2DES. This interpretation is also supported by the absence of the transition involving the B-holes in the PL spectra in the three lowest density samples (Fig. 1), since recombination occurs predominantly with thermalized A-holes in the top valence band states. For the sample $n_{o}=6.5 \times 10^{11} \mathrm{~cm}^{-2}$, a PL peak appears at the same position as the square in the reflectance spectrum; however, this is coincidental. The PL peak is insensitive to $B$ and is therefore ascribed to bulk PL.

The remaining spectral line is marked by circles in Figs. 1 and 4. As opposed to the other spectral lines, it displays a blue shift with increasing density. Upon increasing the density, the confinement potential in the conduction band is expected to narrow down and the subband spacing will go up accordingly. In $\mathrm{MgZnO} / \mathrm{ZnO} \mathrm{HJ}$, this is not only caused by the density increase but also by an enlargement of the conduction band offset with increasing Mg-content. It is therefore natural to attribute this spectral line to non-equilibrium recombination of electrons in the first excited subband $\left(E_{1}\right)$ with photo-excited holes. ${ }^{16}$ The $E_{1}$ line (circle) is well above the $E_{F}$ line (triangle) for all samples, and in PL spectra, the intensity of this line is one order of magnitude weaker than that of the PL-band involving the lowest subband and terminating at $E_{F}$. This assignment would also account for the out-of-phase behavior highlighted in Fig. 2 of these two spectral features. Since the 2DES resides in the lowest subband only, at integer fillings, screening is entirely suppressed and the probability for recombination of electrons excited up into the second subband with valence band states is highest. The PL signal reaches its maximum and weakens again away from integer filling due to screening of the attraction between the

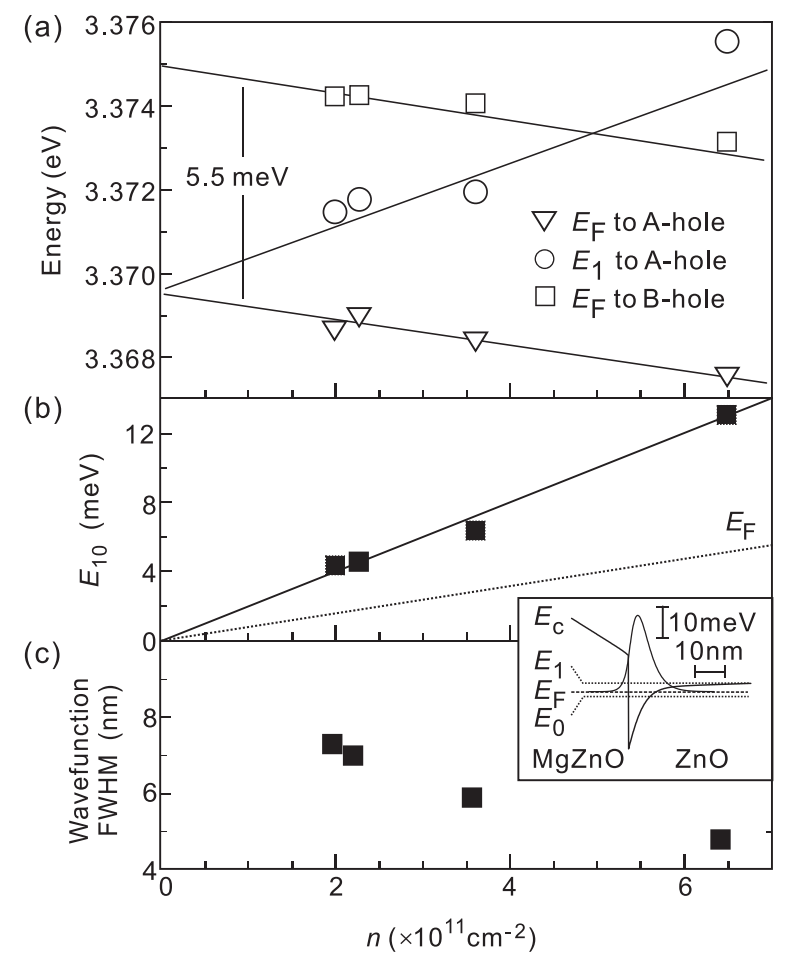

FIG. 4. (a) Density dependence of the 2DES-related spectral features from Fig. 1. The spectral lines marked by triangles and squares are split by $5.5 \mathrm{meV}$, while exhibiting the same red-shift. They are attributed to optical recombination of electrons near $E_{F}$ in the lowest electron subband to holes residing in the A- and B-branch of the valence bands. The circles correspond to a blue-shifting optical transition from the A-valence band branch to the first excited electron subband. (b) The estimated intersubband splitting $\left(E_{10}\right)$ between the lowest and the first excited subband (solid line) and the estimated $E_{F}$ (dotted line). (c) Full-width half-maximum of the 2DES wavefunction at the heterointerface. The inset shows the wavefunction for $n_{o}=2.0 \times 10^{11} \mathrm{~cm}^{-2}, x=0.014$.

photo-excited electrons and holes. To ensure that for the highest density sample the $E_{1}$ spectral feature is properly assigned, it is verified whether the photoluminescence intensities of the lines at $E_{F}$ and $E_{1}$ oscillate anti-phase in a $1 / B$-periodic fashion as for all other samples. Further supporting evidence for proper assignment is obtained by tracing the behavior of the optical transitions as in Fig. 4(a) with density. We conclude that the spectral line marked by circles corresponds to the recombination of electrons from the first excited electron subband and holes in the A-branch of the valence band.

From Fig. 4(a), it follows that at all densities, the second subband is located above $E_{F}$ of the $2 \mathrm{D}$ electrons and it is possible to estimate the energy splitting between the bottom of the second subband, $E_{1}$, and the $E_{F}$ high energy edge. $E_{F}$ itself can be estimated from the density using the band mass ${ }^{17}$ value $m^{*}=0.3 m_{0}$. Combining this information yields an estimate of the intersubband splitting $E_{10}$. It is plotted as a function of density in Fig. 4(b) and exhibits a linear dependence. For the sake of completeness, we note that intersubband splittings extracted from electron-hole transition energies may include corrections from excitonic binding energies, as previously observed in GaAs-based heterostructures with empty excited subbands. ${ }^{16}$ Hence, the data in Fig. 4(b) somewhat underestimate the single-particle splitting $E_{10}$.

Finally, we utilize the experimentally extracted $E_{10}$ and $n_{o}$ to guide calculations of the wavefunctions confined at the 
heterointerface. Using the surface potential of $\mathrm{MgZnO}$ and $\mathrm{ZnO}$ as adjustable parameters, the Schrödinger-Poisson equation is solved self-consistently so that the calculated energy levels $E_{0}$ and $E_{1}$ agree with the experimentally quantified values. A representative wavefunction for sample 282 $\left(n=2.0 \times 10^{11} \mathrm{~cm}^{-2}\right)$ is shown in the inset to Fig. 4. The calculations in panel $\mathrm{c}$ suggest that across the charge density range studied, the full-width half-maximum (FWHM) of the wavefunction is less than $10 \mathrm{~nm}$, in agreement with previous experiments and theoretical calculations. ${ }^{8,18,19}$

In summary, low temperature PL and reflectance spectra of $\mathrm{Mg}_{x} \mathrm{Zn}_{1-x} \mathrm{O} / \mathrm{ZnO} \mathrm{HJ}$ reveal several interband optical transitions connected with the lowest two 2DES subbands and the A- and B-valence bands. In the studied 2DES density range of $2.0-6.5 \times 10^{11} \mathrm{~cm}^{-2}$, the first excited electron subband was shown to lie above $E_{F}$ of the $2 \mathrm{DES}$, and therefore, electrons reside only in the lowest subband. The intersubband energy splitting $E_{10}$ has been estimated in this range of electron densities and grows linearly with increasing density, or alternatively, Mg content. The FWHM of the 2DES wavefunction varies between 8 and $4 \mathrm{~nm}$.

V.V.S., A.B.V., and I.V.K. thank the Russian Scientific Foundation (Grant No.14-12-00693) for support in developing reflectance measurements on $\mathrm{ZnO}$ heterostructures. This work was partly supported by Grant-in-Aids for Scientific Research (S) No. 24226002 from MEXT, Japan, "Funding Program for World-Leading Innovative R\&D on Science and Technology (FIRST)," Program from the Japan Society for the Promotion of Science (JSPS) initiated by the Council for Science and Technology Policy.
${ }^{1}$ L. Schmidt-Mende and J. L. MacManus-Driscoll, Mater. Today 10, 40 (2007).

${ }^{2}$ Y. Kozuka, A. Tsukazaki, and M. Kawasaki, Appl. Phys. Rev. 1, 011303 (2014).

${ }^{3}$ J. Falson, D. Maryenko, Y. Kozuka, A. Tsukazaki, and M. Kawasaki, Appl. Phys. Express 4, 091101 (2011).

${ }^{4}$ A. Tsukazaki, S. Akasaka, K. Nakahara, Y. Ohno, H. Ohno, D. Maryenko, A. Ohtomo, and M. Kawasaki, Nat. Mater. 9, 889 (2010).

${ }^{5}$ D. Maryenko, J. Falson, Y. Kozuka, A. Tsukazaki, M. Onoda, H. Aoki, and M. Kawasaki, Phys. Rev. Lett. 108, 186803 (2012).

${ }^{6}$ E. F. Shubert and K. Ploog, IEEE Trans. Electron Devices 32, 1868 (1985).

${ }^{7}$ E. F. Shubert, A. Fisher, and K. Ploog, Phys. Rev. B 31, 7937 (1985).

${ }^{8}$ J. Betancourt, J. J. Saavedra-Arias, J. D. Burton, Y. Ishikawa, E. Y. Tsymbal, and J. P. Velev, Phys. Rev. B 88, 085418 (2013).

${ }^{9}$ T. Makino, Y. Segawa, A. Tsukazaki, H. Saito, S. Takeyama, S. Akasaka, K. Nakahara, and M. Kawasaki, Phys. Rev. B 87, 085312 (2013).

${ }^{10}$ H. Chen, S. Gu, J. Liu, J. Ye, K. Tang, S. Zhu, and Y. Zheng, Appl. Phys. Lett. 99, 211906 (2011).

${ }^{11}$ I. V. Kukushkin and V. B. Timofeev, Adv. Phys. 45, 147 (1996).

${ }^{12}$ Further details of PL and reflectance spectra will be published elsewhere.

${ }^{13}$ B. K. Meyer, Zinc Oxide: From Fundamental Properties Towards Novel Applications (Springer, The Netherlands, 2005), Chap IV, and references therein.

${ }^{14}$ B. K. Meyer, H. Alves, D. M. Hofmann, W. Kriegseis, D. Forster, F. Bertram, J. Christen, A. Hoffmann, M. Straßburg, M. Dworzak et al., Phys. Status Solidi B 241, 231 (2004).

${ }^{15}$ D. C. Reynolds, D. C. Look, B. Jogai, C. W. Litton, G. Cantwell, and W. C. Harsch, Phys. Rev. B 60, 2340 (1999).

${ }^{16}$ A. J. Turberfield, S. R. Haynes, P. A. Wright, R. A. Ford, R. G. Clark, J. F. Ryan, J. J. Harris, and C. T. Foxon, Phys. Rev. Lett. 65, 637 (1990).

${ }^{17}$ Y. Kasahara, Y. Oshima, J. Falson, Y. Kozuka, A. Tsukazaki, M. Kawasaki, and Y. Iwasa, Phys. Rev. Lett. 109, 246401 (2012).

${ }^{18}$ P. Wang, L. Guo, Z. Song, Y. Yang, T. Shang, J. Li, F. Huang, and Q. Zheng, Physica E 54, 341 (2013).

${ }^{19}$ M. Nakano, A. Tsukazaki, A. Ohtomo, K. Ueno, S. Akasaka, H. Yuji, K. Nakahara, T. Fukumura, and M. Kawasaki, Adv. Mater. 22, 876 (2010). 